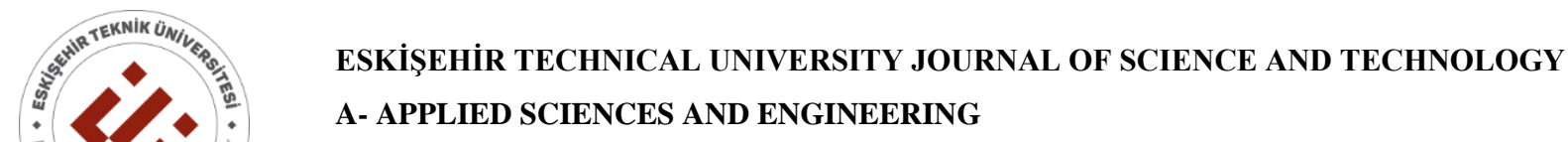

2020, Vol: 21, pp. 17-19, DOI: $10.18038 /$ estubtda. 818009

\title{
INVESTIGATION OF ION EXCHANGE AND MAGNETIC PROPERTIES OF MAGNETICALLY MODIFIED ZEOLITE 13X
}

\author{
Zafer DİKMEN * (iD) \\ Physics Department, Faculty of Science, Eskisehir Technical University, 26470 Eskisehir, Turkey
}

\begin{abstract}
The papers on magnetic modification processes were firstly published about synthetic zeolites and studies continue Petridis et al [1], Čapek et al [2]. The literature review, which we have done up to now, shows that there is no study about the magnetic modification process for $13 \mathrm{X}$ zeolite or that we haven't found one even if there were one. For this reason, this study was realized on magnetic modified $13 \mathrm{X}$ zeolite about the magnetical modification by using magnetite obtained from Divriği region, Turkey. After the modification process, samples were characterized by XRD, XRF, SEM, EDX, VSM. Then, ion exchange and magnetic properties of unmodified and modified zeolites were compared with each other. According to these findings, modified zeolites have better ion exchange and magnetic properties than the others.
\end{abstract}

Keywords: MMZ, 13X Zeolite, Ion exchange, Magnetite

\section{INTRODUCTION}

Magnetically modified zeolites (MMZ) offer the advantage of easy separation and recovery of powdered zeolites after catalytic runs or after environmental remediation using an applied magnetic field. Also, magnetic zeolites could be advantageous in technological processes, including fluidized beds of ferromagnetic particles, ferromagnetic ion exchangers, magnetic refrigeration, and biosciences. There are two methods to obtain magnetically modified zeolites. The first refers to the decoration of zeolite surfaces with magnetic metallic nanoparticles (Fe, Co, Ni). It comprises feeding of zeolites with transition metal ions via ion exchange, followed by reduction of the cations to their metallic state by either hydrogen under drastic conditions or borohydride at ambient temperature. The second involves the modification of natural zeolites with colloidal magnetite particles as described in a patent and briefly reported. In this study, using this novel method, nanoparticles of magnetite which is a mineral $\left(\mathrm{Fe}_{3} \mathrm{O}_{4}\right)$ that is an oxide of iron were settled on the surfaces of $13 \mathrm{X}$ zeolite particles.

\section{EXPERIMENTAL}

In this study, magnetic modified forms of $13 \mathrm{X}$ zeolite, have been prepared, and then the characterization processes such as characterized using methods such as XRD, XRF, SEM, EDX. The ion exchange, adsorption, and magnetic properties of these samples have been investigated. The magnetic modified forms of 13X zeolite samples have been prepared in the following way: The zeolite samples were mixed with magnetite in a mortar and intensively ground into powder in weighty proportion, which is the weight of magnetite divided by the weight of $13 \mathrm{X}$ zeolite and whose magnitude is 3 . The resulting powder mixture was slowly heated up to $550^{\circ} \mathrm{C}$ at $1{ }^{\circ} \mathrm{C} / \mathrm{min}$ and maintained at this temperature for 6 hours. The solid sample was cooled and washed with de-ionized water until no $\mathrm{Cl}^{-}$ions were detected in the eluted water. The mixture solid sample was then dried at $80^{\circ} \mathrm{C}$ for 16 hours Čapek et al [2]. After the modification process, according to XRF analysis, modified samples consist of magnetite $\left(\mathrm{Fe}_{2} \mathrm{O}_{3}\right)$ in a ratio of $63.6 \%$. According to the XRD analysis of the modified sample, magnetite has a maximum

*Corresponding Author: zdikmen@eskisehir.edu.tr

Received: 29.10.2020 Published:27.11.2020 
intensity-peak. SEM images of the modified zeolite sample show us the magnetite nanoparticles on the surfaces of 13X zeolite particles (Figure 1a). According to the EDX result obtained from SEM images for the marked and white region, the surface of the particle consists of an iron in the ratio of $34.5 \%$ weighty. Figure 2 shows that magnetically modified $13 \mathrm{X}$ zeolites exhibit ferromagnetic behavior. CEC of pure zeolite and MMZ that set with the exchange of $\mathrm{Na}^{+}$and $\mathrm{K}^{+}$cations have been calculated by using results of concentration measurements that have been done with Perkin Elmer Optical Emission Spectrometer Optima 4300 DV ICP machine. The cation exchange capacities (CEC) of modified 13X zeolite, unmodified 13X zeolite (Figure 1b) are obtained as $4.94 \mathrm{meg} / \mathrm{g}, 3.86 \mathrm{meg} / \mathrm{g}$, respectively.

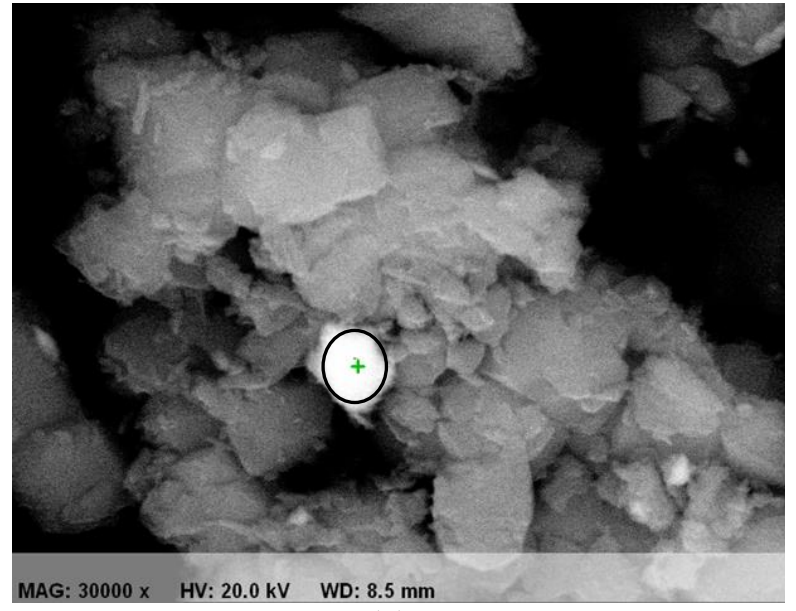

(a)

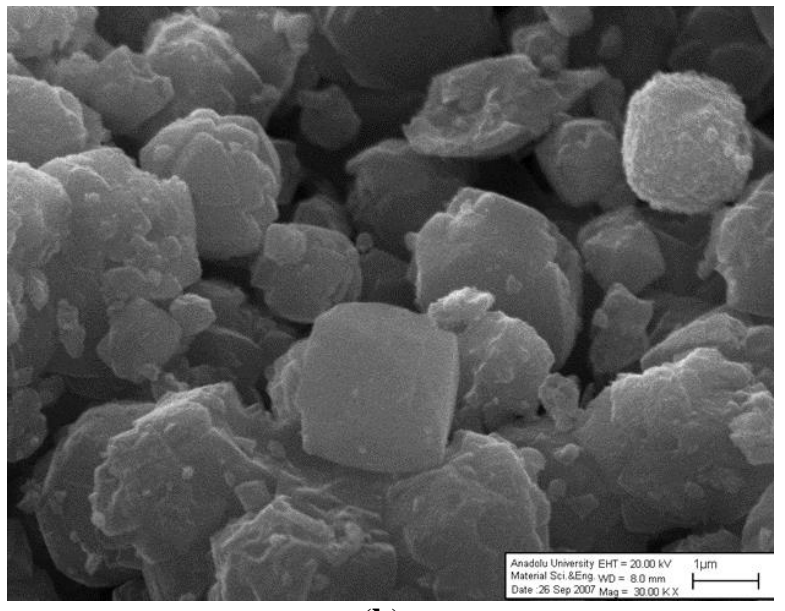

(b)

Figure 1. (a) SEM image of magnetically modified 13X zeolite (b) SEM image of pure 13X zeolite

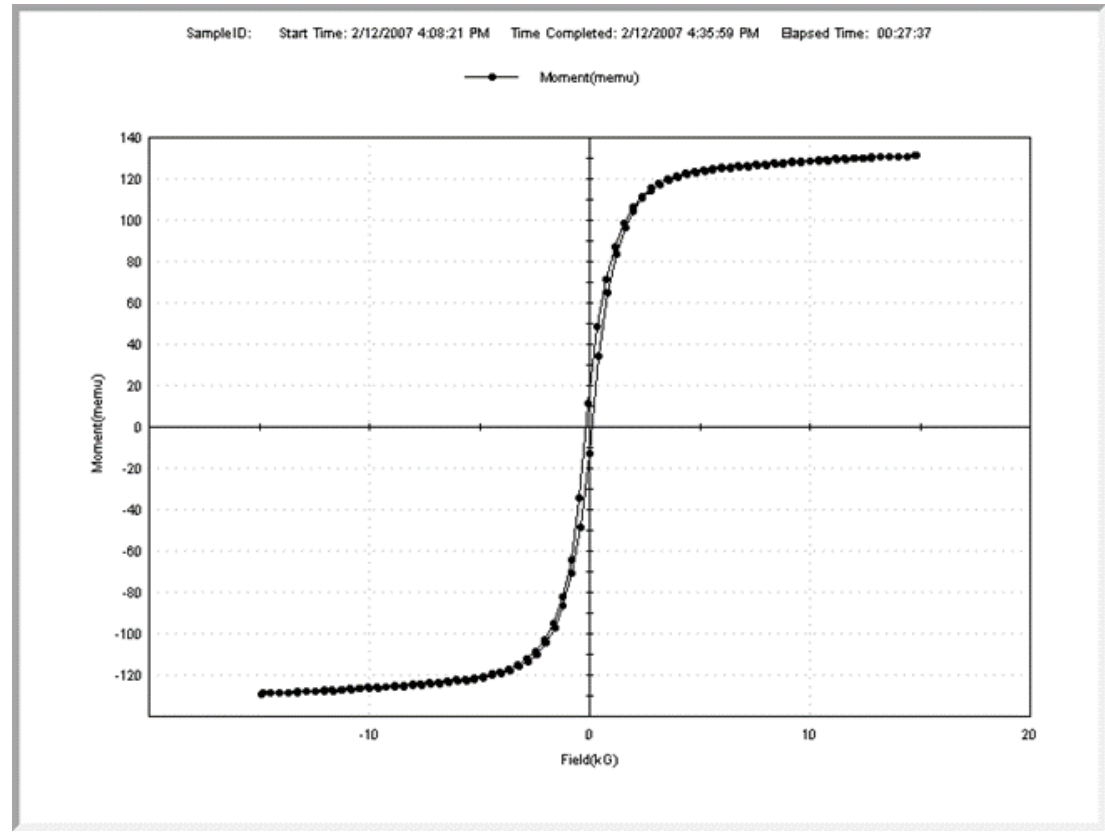

Figure 2. Magnetization versus applied field curve at room temperature for the magnetic modified 13X zeolite. 


\section{RESULTS AND DISCUSSIONS}

The cation exchange capacities (CEC) of magnetic modified 13X zeolite and unmodified 13X zeolite are obtained as $4.94 \mathrm{meg} / \mathrm{g}, 3.86 \mathrm{meg} / \mathrm{g}$, respectively. According to these results, the CEC of magnetically modified zeolite is greater than the CEC of unmodified zeolite in the ratio of \% 27.9 . Figure 1 presents scanning electron micrographs (SEM) of MMZ and pure zeolite, respectively. Figure 2 shows that the bulk magnetization of magnetically modified zeolite is 130 memu and MMZ exhibit ferromagnetic behavior.

\section{REFERENCES}

[1] Petridis D, Bourlinos BA and Zboril R. A simple route towards magnetically modified zeolites Microporous and Mesoporous Materials (2003); 58 (2), 155-162.

[2] Čapek L, Kreibich V, Dědeček J, Grygar T, Wichterlová B, Sobalík Z, Martens JA, Brosius R. and Tokarová V. Analysis of Fe species in zeolites by UV-VIS-NIR, IR spectra and voltammetry. Effect of preparation, Fe loading and zeolite type Microporous and Mesoporous Materials (2005); $80,(1-3), 279-289$. 